\title{
TI.19.1
}

\section{Trusted Delegation of Privileges in an N-Tier Environment (DRAFT 02)}

- PDF: 00018-TrustedDelegationOfPrivilegesInAnNTierEnvironment.pdf

- HTML: draft-lajoie-trust_and_delegation-02.html

More Information

\begin{tabular}{|c|c|}
\hline Repository ID & TI.19.1 \\
\hline Persistent URL & http://doi.org/10.26869/TI.19.1 \\
\hline Title & Trusted Delegation of Privileges in an N-Tier Environment (DRAFT 02) \\
\hline Authors & Chad La Joie, Russell Tokuyama, MACE-WebISO \\
\hline Sponsor & MACE \\
\hline \multicolumn{2}{|l|}{ Review } \\
\hline Status & Legacy \\
\hline Publish Date & $7 / 28 / 2002$ \\
\hline DOI & 10.26869/TI.19.1 \\
\hline \multicolumn{2}{|l|}{ Signature } \\
\hline Deprecated & No \\
\hline \multicolumn{2}{|l|}{ Future Review } \\
\hline \multicolumn{2}{|l|}{ Supersedes } \\
\hline Format & PDF \\
\hline \multicolumn{2}{|l|}{ Related Docs } \\
\hline \multicolumn{2}{|l|}{ Development Location } \\
\hline \multicolumn{2}{|l|}{ IP Framework } \\
\hline Subject Tags & middlewarerescue \\
\hline Notes & \\
\hline
\end{tabular}

